\title{
Deeply Virtual Compton Scattering off the Neutron with CLAS12 at Jefferson Lab
}

\section{Adam HOBART ${ }^{a, *}$ for the CLAS Collaboration}

${ }^{a}$ Laboratoire de Physique des 2 infinis Irène Joliot-Curie - IJCLab, Orsay, France

E-mail: ajhobart@jlab.org

\begin{abstract}
A key step towards a better understanding of nucleon structure in terms of generalized parton distributions (GPDs) is the measurement of Deeply Virtual Compton Scattering off the neutron (nDVCS; $e d \rightarrow e^{\prime} n^{\prime} \gamma(p)$ ). This process allows access to the GPD $E$ of the neutron, which is one of the least constrained GPDs. The determination of $E$, together with $H$ for both the proton and the neutron, can provide information on the quark total angular momentum - via the Ji's sum rule - conveying a more complete picture of nucleon structure. The GPD $E$ is accessed in nDVCS through a Beam Spin Asymmetry (BSA) observable. The measurement of the BSA of nDVCS, combined with observables from pDVCS measurements, allows us to perform the flavor separation of relevant quark GPDs via linear combinations of proton and neutron GPDs. Here, we provide details on the data analysis along with preliminary BSA for nDVCS extracted with the experiment recently carried out at Jefferson Lab using the upgraded CEBAF $11 \mathrm{GeV}$ polarized electron beam and the Hall-B CLAS12 detector.
\end{abstract}

Particles and Nuclei International Conference - PANIC2021

5 - 10 September, 2021

Online

${ }^{*}$ Speaker 


\section{Introduction}

Generalized Parton Distributions [1-8] can provide key information in understanding the nucleon structure. The GPDs describe the correlations between the longitudinal momentum and transverse spatial position of the partons inside the nucleon $[1,4,7,8]$, they give access to the contribution of the orbital momentum of the quarks to the nucleon spin [2,3], and they are sensitive to the correlated $q-\bar{q}$ components [5, 6, 8]. The nucleon GPDs are accessed in the experiments of exclusive lepto-production of a photon (e.g. Deeply Virtual Compton Scattering - DVCS process), or of a meson (e.g. Deeply Virtual Meson Production) off the nucleon at sufficiently large $Q^{2}$, where $Q^{2}$ is the virtuality of the exchanged photon.

Considering only helicity-conserving quantities and the quark sector, there are four GPDs, $H, \tilde{H}, E, \tilde{E}$, which depend, in leadingorder and leading-twist $\mathrm{QCD}$, upon three variables: $x, \xi$ and $t$ (see [1] for more details). The quantities $x-\xi$ and $x+\xi$ are the longitudinal momentum fractions of the quarks coming out and going back into the nucleon, respectively and $t$ is the squared fourmomentum transfer between the final and initial nucleon. Figure 1 illustrates the leading-order Feynman diagram for DVCS and the definitions of the relevant kinematic variables.

In an experimental analysis, the DVCS process, which naturally

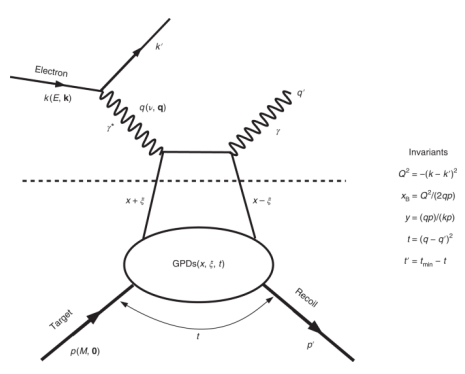

Figure 1: The handbag diagram for DVCS. interferes with the Bethe-Heitler $(\mathrm{BH})$ process where the final state photon is emitted by the lepton, allows access to combinations of GPD-related quantities called the Compton Form Factors $\operatorname{CFF}(\xi, t)$. In an experimental configuration where the beam is polarised and the target is unpolarised, one of the accessible observables is the Beam Spin Asymmetry (BSA) which relates to the $C F F$ as

$$
A_{L U} \propto \sin (\Phi) \mathfrak{J}\left(F_{1} \mathcal{H}+\xi\left(F_{1}+F_{2}\right) \mathcal{H}+-k F_{2} \mathcal{E}+\ldots .\right)
$$

where $\Phi$ is the angle between the lepton scattering and photon production planes, $F_{1}$ and $F_{2}$ are the Dirac and Pauli form factors, and $\mathcal{H}$ and $\mathcal{E}$ are the $C F F$ related to the GPDs $H$ and $E$, respectively.

In this paper, we present the preliminary results and analysis of the BSA of DVCS from a deuterium target (nDVCS, $e d \rightarrow e^{\prime} n^{\prime} \gamma(p)$ ) using the CLAS12 detector [9] at Jefferson Lab. The BSA amplitude is dominated by the $C F F(\mathrm{GPD}) \mathcal{E}(E)$ of the neutron. The GPD $E$ is one of the least known GPDs, and only one previous measurement exists from pioneering experiments performed in Hall-A at Jefferson Lab [10]. However, the experiment reported here is the first measurement with tagging of the active neutron and hence, allowing for a precise determination of the BSA. $E$ is one of the two GPDs that enter into the Ji's sum rule [2,3], which links the total angular momentum $\left(J_{q}\right)$ carried by each quark $q$ to the sum of the GPDs $H$ and $E$.

\section{Experiment and Event Selection}

The experiment has taken place in the Hall-B of JLab utilizing the large acceptance spectrometer CLAS12 [9]. The CEBAF accelerator produces a high-intensity, high-duty-factor, polarized electron beam. An average beam polarization of $\sim 85 \%$ was measured throughout the experiment in dedicated Möeller runs. The target was an unpolarized liquid deuterium cell measuring $5 \mathrm{~cm}$ long.

To guide the data analysis a Monte-Carlo simulation was used. An event generator for incoherent electroproduction of photons on deuterium was adopted, which produces either $e d \rightarrow e^{\prime} n^{\prime} \gamma(p)$ 
or $e d \rightarrow e^{\prime} p^{\prime} \gamma(n)$ events, proportionally to their relative cross-sections, coming from the $\mathrm{nD}$ VCS/pDVCS and BH reactions [11]. The DVCS amplitude is calculated according to the BMK formalism [12]. The Fermi-motion distribution is calculated with the Paris potential [13].

The reaction of interest is $e d \rightarrow e^{\prime} n^{\prime} \gamma(p)$. Events with at least one identified electron, one photon with energy above $2 \mathrm{GeV}$, and one neutron with momentum above $0.35 \mathrm{GeV} / c$ were selected. The particle identification of CLAS12 has been used to identify all particles within each event [14]. In the case where multiple final-state particles of the same type are present in the event, all possible combinations of the exclusive final state were considered for treatment. The best combination was taken to be the one minimizing a multi-dimensional $\chi^{2}$ calculated using variables related to the exclusive final state.

Several cuts were applied in order to select the relevant kinematic region for the $\mathrm{nDVCS}$ reaction. The electron momentum was selected to be above $1 \mathrm{GeV}$. We selected neutron momentum to be above $0.35 \mathrm{GeV} / c$ to remove spectator-neutron events. A cut on the cone angle formed by the electron and the neutron $\left(\theta_{e, N}>5^{\circ}\right)$ was applied to remove radiative photons reconstructed as neutrons. A cut on the cone angle formed by the electron and the photon $\left(\theta_{e, \gamma}>5^{\circ}\right)$ was applied to remove radiative photons that were likely emitted close to the electron. We cut on $Q^{2}>1 \mathrm{GeV}^{2}$ and $W>2 \mathrm{GeV}$ to ensure the applicability of the leading-twist GPD formalism and remove contributions from the resonance region. We cut also on $E_{\gamma}>2 \mathrm{GeV}$ as photons from nDVCS process are expected at high energies. Moreover, fiducial cuts were applied to

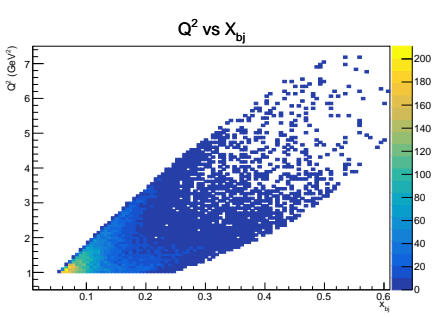

Figure 2: $Q^{2}$ versus $x_{B}$ for the nDVCS data sample with all selection cuts applied. The large kinematic reach of CLAS12 is clearly demonstrated in this distribution. remove regions where the detector acceptance varies rapidly.

Exclusivity cuts were applied to select the $e^{\prime} n^{\prime} \gamma(p)$ final state while minimizing the background. Cuts on the missing mass in the $e d \rightarrow e^{\prime} n^{\prime} \gamma X$, en $\rightarrow e^{\prime} n^{\prime} \gamma X$ and $e n \rightarrow e^{\prime} n^{\prime} X$ reactions and the missing momentum in the $e d \rightarrow e^{\prime} n^{\prime} \gamma X$ reaction were considered. Cuts were also applied on the difference between the angle $\Phi$ between the lepton scattering and photon production planes, computed using the neutron and the virtual photon and using the virtual and the real photon $(\Delta \Phi$ ). Similarly, a cut was applied on the difference between two ways to compute $t$, either using the scattered neutron or using the virtual and real photon $(\Delta t)$. Finally, a cut on the cone angle between the detected and the reconstructed photon from missing particle selection, $\theta_{\gamma, X}$ was applied to reduce the contamination of photons from the partially reconstructed $\pi^{0}$ decay.

Due to inefficiencies in the tracking system of the CLAS12 detector and to the existence of dead regions in the tracker acceptance, some reconstructed neutrons were misidentified protons that were cut out using a multivariate analysis technique (Boosted Decision Trees) and utilizing final state exclusivity variables.

Figure 2 shows the two dimensional plane in $Q^{2}$ and $x_{B}=\frac{Q^{2}}{2 M v}$ (see Fig. 1 for kinematic variables definitions) after all selection cuts are applied. The distribution shows clearly the large kinematic reach that the high acceptance CLAS12 detector can provide for the measurement of asymmetry observables in the DVCS processes. 

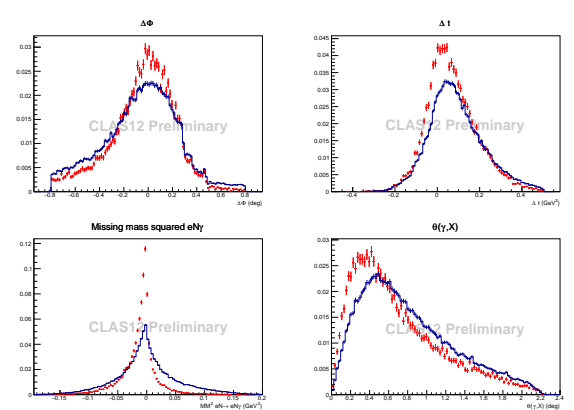

$\theta(y, x)$

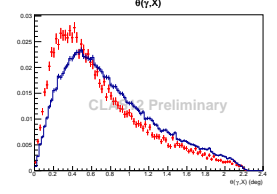

Figure 3: Top left: $\Delta \Phi$. Top right: $\Delta t$. Bottom left: the missing mass in the $e n \rightarrow$ en $X$ reaction. Bottom right: the cone angle between the detected and the reconstructed photon $\theta_{\gamma, X}$. Data (in blue) contains background from partially reconstructed $\pi^{0} \mathrm{de}-$ cays. Simulations (in red) of DVCS/BH on deuterium containing both the neutron and proton DVCS/BH channels. in data to get the number of $e^{\prime} n^{\prime} \pi^{0}$ (1 photon) in data. This number is subtracted from neutron DVCS reconstructed decays in data for each kinematic bin and it is evaluated to range between $10-45 \%$ depending on the bin in consideration.

\section{Extraction of the Beam Spin Asymmetry Observable}

The BSA, $A_{L U}$, is defined as $A_{L U}=\frac{1}{P} \frac{N^{+}-N^{-}}{N^{+}+N^{-}}$where $P$ is the average beam polarisation and $N^{+(-)}$stands for the number of DVCS events for positive (negative) beam helicity. Figure $4 \mathrm{a}$ shows the distribution of $A_{L U}$ for nDVCS integrated over the whole kinematic domain. Given the available statistics, we performed an extraction of the BSA in two dimensional bins in $Q^{2}, x_{B}$ or $t$ versus $\Phi$. We considered three bins in $Q^{2}[1,1.9]$, [1.9, 2.9], and $[2.9,6] \mathrm{GeV}^{2}$ (Fig. 4b), three bins in $X_{B}$ [0.05, 0.1], [0.1, 0.17], and [0.17, 0.6] (Fig. 4c) and three bins in $-t[0,0.3],[0.3,0.5]$, and $[0.5,1.1] \mathrm{GeV}^{2}$ (Fig. $\left.4 \mathrm{~d}\right)$. The distribution is modeled with the function $(a \sin (\Phi))$ in accordance with Eq. (1).

\section{Conclusions}

We have presented the analysis status of nDVCS experimental data obtained from polarised electron beam scattering off a deuterium target. The analysis exploits the full kinematic coverage of the CLAS12 detector. Therefore, we performed an extraction of the BSA observable in twodimensional bins in $Q^{2}, x_{B}$ or $t$ versus $\Phi$. The systematic uncertainty on the $\pi^{0}$ background subtraction will be evaluated using two different subtraction techniques. The systematic uncertainty related to proton contamination will be evaluated by varying the selection cut from the Boosted Decision Trees. The systematic uncertainty related to the applied exclusivity selection will be estimated by applying different variations of the selection. The systematic uncertainty on the beam polarization will be estimated by checking the standard deviation of the beam polarisation over the Möller runs. We expect that the statistical uncertainty is much larger than the systematic one. The analysis is preliminary and results need to be refined before being confronted to theoretical models. 


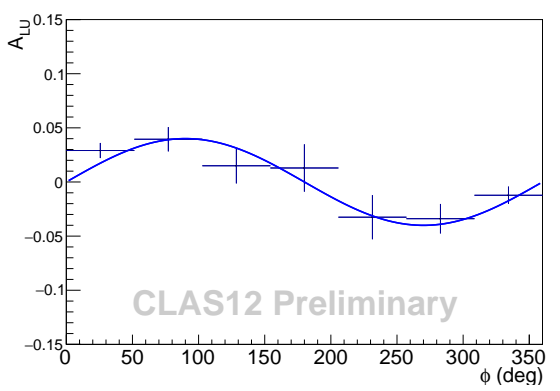

(a) BSA integrated over the whole kinematic domain.
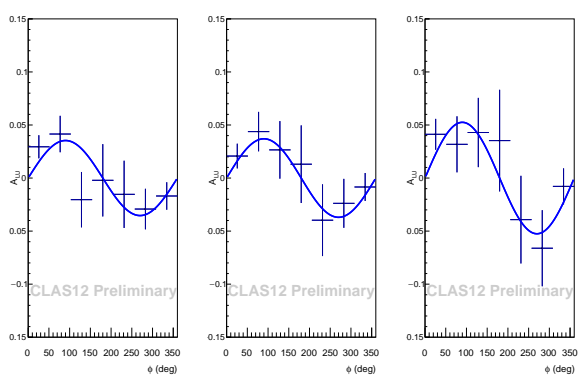

(b) BSA in bins of $Q^{2}$. Left $[1,1.9] \mathrm{GeV}^{2}$, middle $[1.9,2.9]$ $\mathrm{GeV}^{2}$, and right $[2.9,6] \mathrm{GeV}^{2}$.
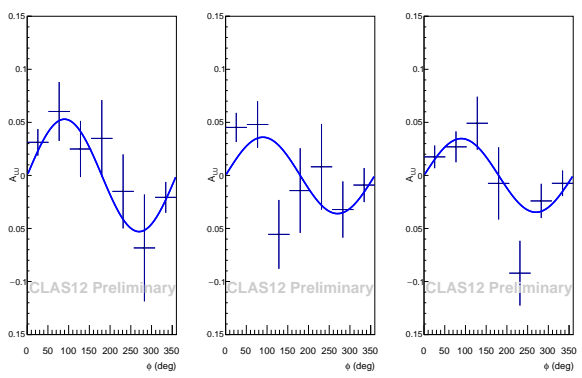

(c) BSA in bins of $x_{B}$. Left [0.05, 0.1], middle [0.1, 0.17], (d) BSA in bins of $t$. Left $[0.5,1.1] \mathrm{GeV}^{2}$, middle [0.3, 0.5] and right $[0.17,0.6]$.

$\mathrm{GeV}^{2}$, and right $[0,0.3] \mathrm{GeV}^{2}$.

Figure 4: Preliminary: nDVCS BSA. Error bars represent statistical uncertainties only.

\section{References}

[1] D. Müller, D. Robaschik, B. Geyer, F.-M. Dittes, and J. Horejsi. Fortschr. Phys. 42 (1994) 101.

[2] X. Ji, Phys. Rev. Lett. 78 (1997) 610

[3] X. Ji, Phys. Rev. D 55 (1997) 7114.

[4] A.V. Radyushkin, Phys. Lett. B 380 (1996) 417; Phys. Rev. D 56 (1997) 5524.

[5] J.C. Collins, L. Frankfurt and M. Strikman, Phys. Rev. D 56 (1997) 2982.

[6] ] K. Goeke, M. V. Polyakov and M. Vanderhaeghen, Prog. Part. Nucl. Phys. 47 (2001) 401.

[7] M. Diehl, Phys. Rept. 388 (2003) 41

[8] A.V. Belitsky, A.V. Radyushkin, Phys. Rept. 418 (2005) 1.

[9] V.D.Burkert et al. NIM Physics A. 959 (2020) 163419.

[10] M. Benali et al. Nature Phys. 16 (2020) 191.

[11] A. El Aloui and E. Voutier, private communication.

[12] A.V. Belitsky, D. Müller, A. Kirchner, Nucl. Phys. B 629 (2002) 323-392. 
[13] M. Lacombe et al., Phys. Rev C 21 (1980) 861.

[14] V.Ziegler et al. NIM Physics A. 959 (2020) 163472. 